\title{
The effects of anode distance and corrosion activity on current distribution for ICCP systems
}

\author{
Shamir Bhuiyan*, David Law, Liam Ward, and Justin Saliba \\ School of Engineering, RMIT University, Melbourne, VIC, 3001, Australia
}

\begin{abstract}
One of the most effective techniques to mitigate corrosion of steel reinforcement in concrete is impressed current cathodic protection (ICCP). This technique has been widely used for several decades; however, there is limited research systematically studying the various factors affecting the distribution of cathodic current over the area of steel reinforcement. Understanding how current is distributed in an ICCP system is crucial for its design to ensure that all areas of steel are sufficiently protected, and overprotection of certain areas is avoided. In this study, the effect of distance of the anode from the steel and level of corrosion on current distribution is investigated by applying a potential sweep and measuring the steel potential and current for specimens with three layers of steel at different depths. The findings from this study revealed an inverse relationship between cathodic polarisation with steel distance from the anode and that higher levels of corrosion can cause a more non-uniform current distribution favouring the closest bar to the anode.
\end{abstract}

\section{Introduction}

One of the key considerations in designing an impressed current cathodic protection system (ICCP) is the aspect of current distribution due to its non-uniform nature in most practical scenarios. In the context of reinforced concrete structures, it is critical to ensure that the total area of steel bars receives an adequate level of current for protection whilst avoiding overprotection of any areas. Current distribution is associated with throwing power which is the ability to polarize the steel at different depths, which in turn depends on a number of factors, namely magnitude of the supplied current, concrete resistance, corrosion rate and reinforcement geometry/arrangement [1-3].

Concrete resistance is a function of the material property (ie resistivity) and the physical dimensions of the reinforced concrete area. In the context of this study, the former is kept constant while the latter is varied by changing the anode-steel distance. Following Ohm's law, a path of greater resistance will result in a lower current for a given driving voltage, resulting in lower polarization. Consequently, the amount of current received is dependent on the position of the bars in relation to the anode. In a study conducted by Bertolini et al [2], wherein a slab with double-layered reinforcement was cast, it was found that the steel layer closest to the anode received between $70-90 \%$ of the total current, although this included bars which were positioned directly above one another. A similar range of results were reported by $\mathrm{Xu}$ et al [3] and Hassanein et al [1] for their experiments which also involved multi-layered steel reinforcement.

With regards to corrosion rate, this can be correlated directly with the resistance to polarization, with a higher corrosion rate implying a need for a greater amount of current to overcome it. Computer and theoretical models $[2,4]$ have predicted that current requirements can increase several times over in order to provide the same level of polarization for a corroding bar compared to a passive bar. For concrete with actively corroding steel, the current distribution tends to be more uneven and favours bars closer to the anode, provided corrosion rates do not vary in the structure $[1,3]$. In cases where an activepassive couple exists, the current distribution is further complicated [5].

The objective of this study is to demonstrate the effects of anode distance and resultant corrosion rates on the level of polarization of the bars to develop an understanding of how current is distributed within three layers of reinforcement by applying a potentiodynamic scan. This paper reports the initial ( 0 and 5 months data) results to assess the effect of bar position of current distribution. This specifically addresses the period during the initiation of corrosion.

\section{Methodology}

In order to study the effect of distance of anode from the steel reinforcement on the level of cathodic polarisation, a simple setup was designed, wherein 3 steel reinforcement bars and a platinized titanium ribbon (anode) were cast in a concrete block measuring $350 \times 100 \times 100 \mathrm{~mm}$, with a design configuration as shown in figure 1a. The anode was placed above the bars in order to study the effect of distance alone. For reference purposes, the closest steel bar to the anode was named bar 1 and the second closest and furthest, bar 2 and bar 3 respectively. The horizontal spacing between the anode

Corresponding author: Shamir.bhuiyan@ @rmit.edu.au 
and bars 1, 2 and 3 was $25 \mathrm{~mm}, 125 \mathrm{~mm}$ and $225 \mathrm{~mm}$ respectively whereas the vertical spacing between the anode and centreline of the bars was $50 \mathrm{~mm}$. The actual reinforced concrete test setup prior to and after casting is shown in figures $1 \mathrm{~b}$ and $1 \mathrm{c}$ respectively.
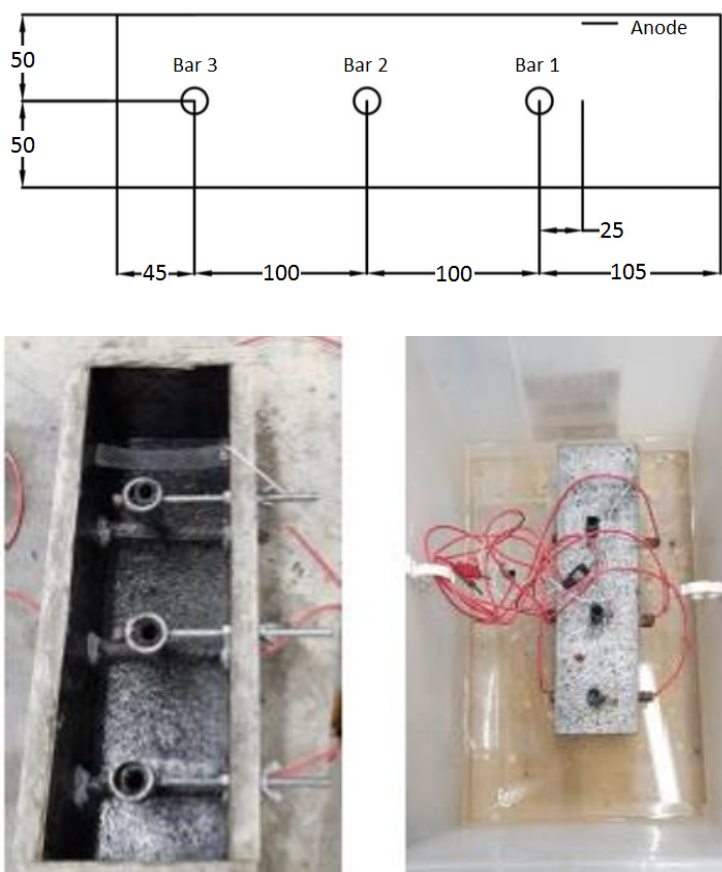

Fig. 1a-c. Specimen layout

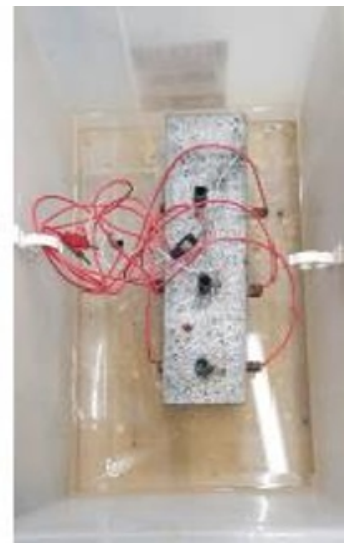

The bars were $13 \mathrm{~mm}$ diameter mild steel and spanned the width of the mould, as did the anode ribbon having a width of $20 \mathrm{~mm} . \mathrm{Cu} / \mathrm{CuSO}_{4}$ electrodes were utilized as reference electrodes and were positioned directly above each bar. The housings of the electrodes were made from PVC tubing which were plugged at the ends with porous wooden stoppers. Before each measurement, the tubes were filled with $\mathrm{CuSO}_{4}$ solution and $\mathrm{Cu}$ wires were inserted into the solution. The concrete mix design, table 1, was a standard $40 \mathrm{MPa}$ mix, using General Purpose (GP) cement. To study the effects of corrosion level on current distribution, $5 \% \mathrm{NaCl}(3.5 \% \mathrm{Cl}$ ion) by weight of cement was added to the wet mix to initiate corrosion of the bars which would allow the corrosion levels to vary over time. The specimen was periodically cycled with water (once per week) and stored in a sealed container to maintain a high-humidity environment to promote corrosion.

Table 1. Concrete mix design

\begin{tabular}{|l|c|}
\hline \multicolumn{1}{|c|}{ Constituents } & Quantity $\left(\mathrm{Kg} / \mathrm{m}^{3}\right)$ \\
\hline (GP) Cement & 390 \\
\hline Water $(\mathrm{w} / \mathrm{c:} 0.65)$ & 254 \\
\hline Fine Aggregate & 585 \\
\hline Coarse Aggregate $(7 \mathrm{~mm})$ & 585 \\
\hline Coarse Aggregate $(10 \mathrm{~mm})$ & 585 \\
\hline
\end{tabular}

After casting the concrete, an initial set of readings was taken within 48 hours representing the initial corrosion initiation period, when passivation and corrosion initiation where occurring. A second set of readings was taken after a 5 month period by which point in time active corrosion was established. Potentiodynamic cathodic polarization scans were conducted in order to determine the current distribution in the system. Scans were conducted at a rate of $100 \mathrm{mV} / \mathrm{min}$ from the rest potential to $-800 \mathrm{mV}$ (corresponding to bar1) using a potentiostat (ACM Field Machine). This corresponded to a period of between 3-5 minutes depending upon the rest potential. The anode was connected as the auxiliary electrode, the steel bars as the working electrodes and the $\mathrm{Cu} / \mathrm{CuSO}_{4}$ electrode (bar1) as the reference electrode for operating the potentiostat. During the scans, the potentials and currents for each bar were recorded using a data-logger (DataTaker DT80). In order to measure the currents received by each bar, the bars were connected to $100 \Omega$ resistors in series and the voltage measurements across the resistors were logged. A multiplier of 0.01 was applied to convert voltage readings to amps. The wiring diagram is shown in figure 2.

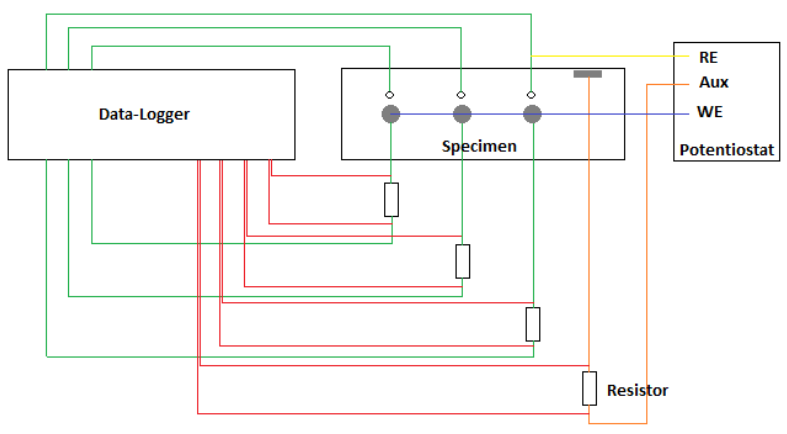

Fig. 2. Wiring diagram of experimental setup

\section{Results}

\subsection{Data at $48 \mathrm{~h}$ (0 month)}

Variations in bar current with anode current (total applied current measured across the anode) are shown in figure 3. Results were plotted up to $0.5 \mathrm{~mA}$ anode current, which corresponded to a current density of approximately 120 $\mathrm{mA} / \mathrm{m}^{2}$. Figure 4 shows the variation in potential for the three bars with anode current. As expected, bar 1 being closest to the anode received the greatest portion of the current (figure 3 ) and was thus polarized the most (figure 4). Bars 2 and 3 received considerably lower current, and thus the extent of polarisation was reduced accordingly. Additionally, bar 3 appeared to receive a higher current than bars 1 and 2 until $0.08 \mathrm{~mA}$ and $0.37 \mathrm{~mA}$ anode current respectively, despite it being further away from the anode.

It should be noted that there was an offset of the bar current for bars 2 and 3 at the beginning of the scan which could be due to a small macro-cell current flowing between them through the system. This is supported by the difference in the rest potential of bar 3 as compared to bars 1 and 2 (figure 4). The presence of this potential difference could account for the current flow observed. 
Further analysis of the anode - bar current plots in figure 3 reveal the rate of change of bar current with respect to the anode current decreases with the anode-steel distance (ie highest in bar 1 and lowest in bar 3). These observations in slope variation suggest that although bar 3 appears to initially receive greater current than bars 1 and 2 , it actually receives less of the cathodic current throughout the scan. These findings suggest that as the overall anode current is increased, a greater proportion of this current is received by the nearest bar and smaller proportions by the bars further away. This is important when considering overall design of cathodic protection systems in terms of receiving sufficient protective current by bars further away from the anode.

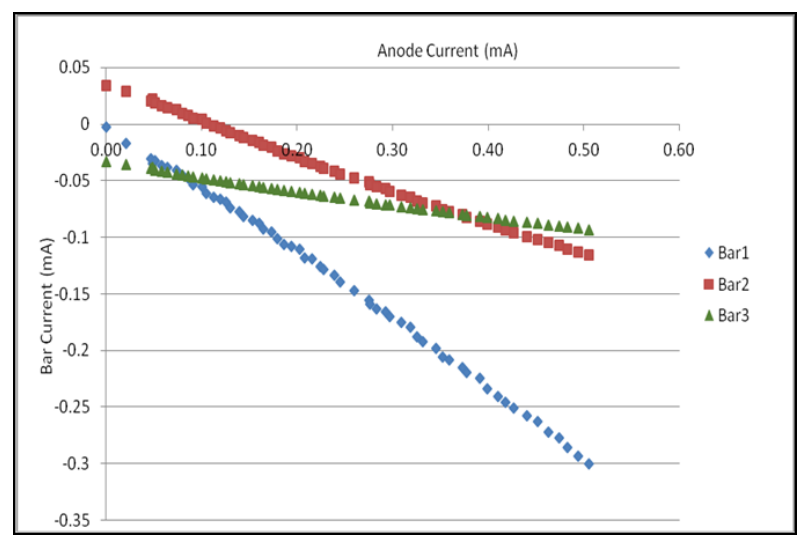

Fig. 3. Bar current vs anode current for 0 month

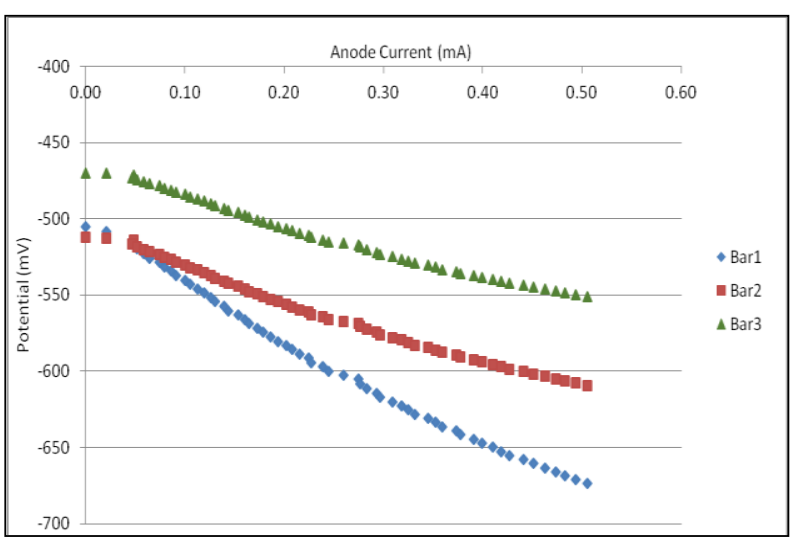

Fig. 4. Potential vs anode current for 0 month

\subsection{Data after 5 months}

Variations in bar current with anode current after 5 months is shown in figure 5. Similar trends were observed as for 0 month data, except that a greater proportion of current was received by the nearest bar, particularly at the higher anode currents (-0.3 mA for bar 1 for $0.5 \mathrm{~mA}$ anode current at 0 months, compared with $-0.35 \mathrm{~mA}$ after 5 months). Figure 6 shows the variation in potential for the three bars with anode current after 5 months. Here, due to increased corrosion activity, the rest potentials of all bars had shifted in the negative direction, ranging between 550 and $-600 \mathrm{mV}$. The rest potentials of all the bars were also closer together than the 0 month data, suggesting a more uniform corrosion state of the bars had been established after 5 months. The data indicates that the macrocell current was still evident, however to a lesser degree, as seen from their trends in figure 5. Bar 3 no longer showed a higher initial bar current than bar 1 and the point up till which bar 3 displayed a higher bar current than bar 2 had also receded to $0.18 \mathrm{~mA}$ (anode current).

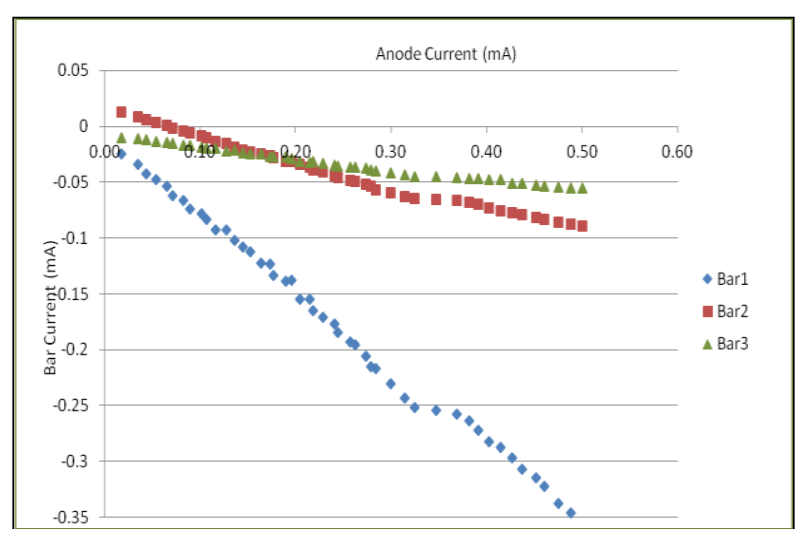

Fig. 5. Bar current vs anode current for 5 months

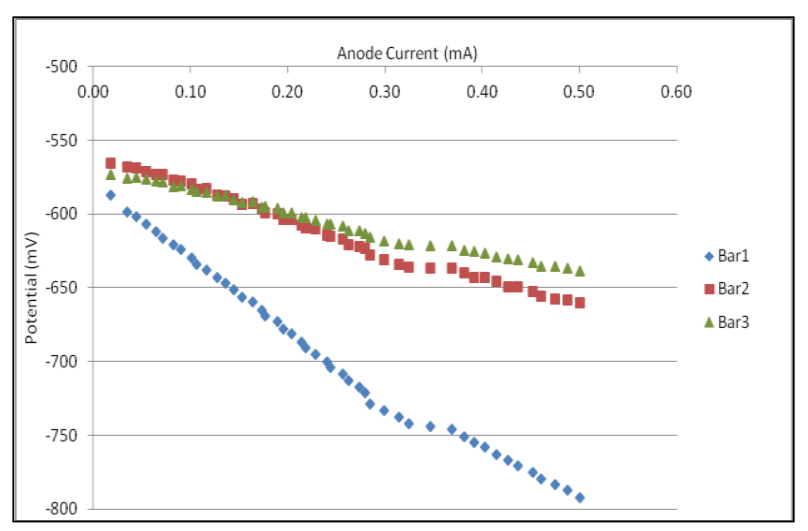

Fig. 6. Potential vs anode current for 5 months

\subsection{Discussion}

In order to compare and analyse the data, the 0 month and 5 months polarisation data (anode versus bar currents) have been combined and plotted in a bar chart, as shown in figure 7. Polarization over-potential, calculated as the difference between the open circuit potential and polarized potential, versus anode current data is plotted in a bar chart, figure 8 . It is clearly observed that bar 1 received the greatest portion of the cathodic current for both data sets. For the 0 month scan, bar 1 received an average of approximately $55 \%$ of total current received across all the bars whereas for the 5 months scan, this percentage increased to $70 \%$. This is in agreement with previous research [1-4]. The variation in current distribution between 0 month and 5 months is attributed to the establishment of corrosion of the bars. At 0 months, the bars were subject to both a passive state due to the hydration reaction of the cement and active state, due to corrosion initiation by the cast-in chlorides. At this point, corrosion rates tend to be sporadic [6] and it can be observed that bar 3 at 0 month had a different rest potential compared to bars 1 and 2 , thus indicating a difference in corrosion rate After 5 months exposure, a 
similar degree of corrosion had been established on all bars, as indicated by the more negative, but similar potentials observed.

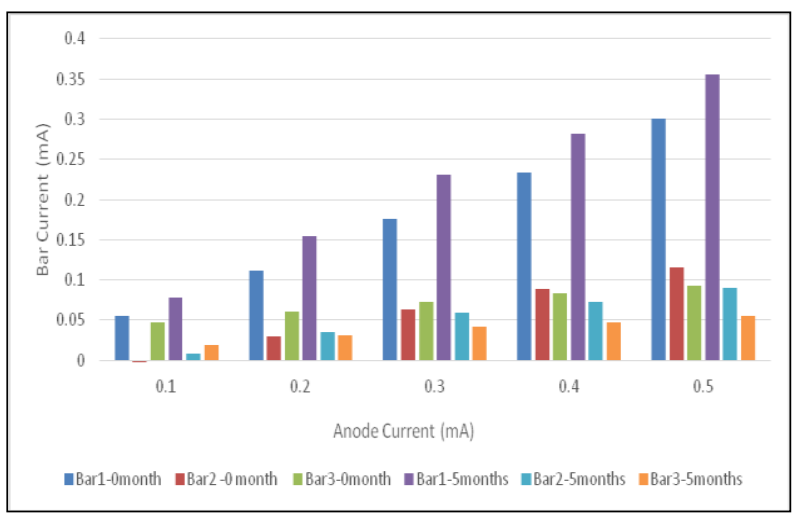

Fig. 7. Comparison of individual absolute bar currents at fixed anode currents

Figure 8 shows polarization trends were consistent, with bar 1 being polarized the most and bar 3 the least. The polarization trends generally followed the same trends as the bar current, i.e. increasing overpotential with increasing anode current. This is expected given that they are directly related. However, for $0.1-0.3 \mathrm{~mA}$ anode current data (figure 7) it can be observed that bar 2 appeared to receive lower current than bar 3 although the corresponding data for the polarization in figure 8 shows that bar 2 was consistently polarized to a greater degree. It is hypothesized that the current results were offset due to the formation of an initial macrocell and that the actual current received at the bars was consistent with polarization trends throughout the scan. This is further supported by the gradient variations as observed in figures 3 and 5. In terms of the bar distances from the anode in this study representing varying layers of steel reinforcement, the increase in current being drawn by bar 1 at 5 months compared to 0 month indicates that the current was preferentially drawn to the top steel layer more so when corrosion rates are more uniform and higher at 5 months compared to the initial data where corrosion rates are lower and the corrosion rates vary between the bars.

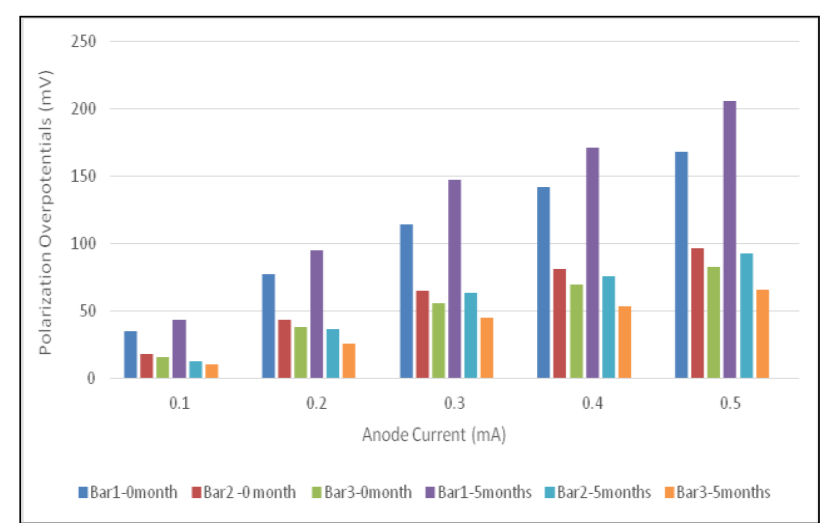

Fig. 8. Comparison of polarization overpotentials at fixed anode currents
Furthermore, the resistivity in the concrete would be expected increase between the initial data and the 5 month data due to hydration of the cement. This could itself lead to a change in the balance between the ohmic resistance and the polarisation resistance and hence the current distribution to the bars.

At 5 months the current distributions across the three bars are in agreement with predicted results in literature $[2,4]$. However, the results do indicate that the specific distribution of the current between the bars is influenced by the condition of the bars and the corrosion that is occurring on each bar relative to the other layers of steel. Thus, there may be an effect of dissimilar corrosion rates on the current distribution and hence the protection afforded to the steel. This may be important in the design of ICCP systems and further tests are required to validate the effect and assess the impact on the protection afforded to the steel.

\section{Conclusions}

Experimental results showed that bar1, which was closest to the anode, received between $55-70 \%$ of the total current, which is similar to findings stated in the literature for long term experiments. The effect of anode-steel distance was demonstrated, wherein a greater distance resulted in a greater path of resistance for the current and thus a lower degree of polarization for a given anode current. The 0 month data showed that bar 1 , closest to the anode, received approximately $55 \%$ of the total current, which increased to $70 \%$ by 5 months. This is attributed to the increase in corrosion rates of the bars and the resitivity in the concrete.

\section{References}

1. A.M. Hassanein, G.K. Glass, N.R. Buenfeld, "Protection current distribution in reinforced concrete cathodic protection systems", Cement \& Concrete Composites 24, 159-167 (2002)

2. L. Bertolini et al, "Cathodic protection of new and old reinforced concrete structures", Corrosion Science 35, 1633-1639 (1993)

3. J. $\mathrm{Xu}, \mathrm{Wu}$ Yao, "Current distribution in reinforced concrete cathodic protection system with conductive mortar overlay anode", Construction and Building Materials 23, 2220-2226 (2009)

4. G.K. Glass, A.M. Hassanein, "Surprisingly Effective Cathodic Protection", Journal of Corrosion Science and Engineering 4, paper7 (2006)

5. A.A. Sagüés, S.C. Kranc, "On the determination of polarisation diagrams of reinforcing steel in concrete", Corrosion Science 48(8), 624-33 (1992)

6. S. Bhuiyan, D.W. Law, P Nicholls, C. Christodoulou, "Investigation of residual protection of steel following application of protective current", Construction and Building Materials 162, 503-511 (2018) 